\title{
Complicaciones en recién nacidos de madres adolescentes tempranas en el Hospital Nacional Arzobispo Loayza de mayo del 2008 a mayo del 2012
}

\author{
Alonso F. La Rosa ${ }^{1,2}$
}

\section{RESUMEN:}

Objetivo: Debido al aumento de la tasa de fecundidad en las adolescentes, el interés por las posibles complicaciones también ha aumentado. Varios estudios concluyen que el embarazo en las edades extremas del periodo reproductivo, son un factor de riesgo para complicaciones en los recién nacidos. Otros estudios no encuentran asociación entre éstas. El objetivo de este trabajo fue analizar la relación de complicaciones en recién nacidos de madres adolescentes tempranas (10-14 años)comparada a los de madres adultas entre 20 a 35 años atendidas en el HNAL.

Material y Métodos: Estudio analítico, tipo cohorte retrospectivo. La población fue el total de recién nacidos de madres adolescentes tempranas y, un número igual de madres entre 20 a 35 años.

Resultados: Del total $(16,601)$ de nacidos, $62(0.37 \%)$ fueron hijos de madres adolescentes tempranas. Sobre el peso al nacer, la mayoría tuvo un peso adecuado (87.1\% y $80.6 \%$ respectivamente). Sobre el peso para la edad gestacional, $85.5 \%$ y $72.6 \%$ fueron adecuados. En edad gestacional, $90.3 \%$ de ambos grupos, fueron a término. Parto vaginal se dio $82.3 \%$ y $69.4 \%$ en cada grupo. $32.3 \%$ y $19.4 \%$ no tuvieron adecuado control prenatal. Ictericia no presentó en $95.2 \%$ y $96.8 \%$ respectivamente. Sepsis en $93.5 \%$ y $96.8 \%$. No Complicación respiratoria en $96.8 \%$ en ambos grupos. Complicaciones mecánicas $90.3 \%$ y $95.2 \%$. Conclusiones: La frecuencia de recién nacidos de madres adolescentes tempranas es menor a la estadística nacional. No existió diferencia entre las complicaciones de los recién nacidos de madres adolescentes tempranas en relación a los de madres entre 20 a 35 años. (Horiz Med 2015; 15(1): 14-20)

Palabras clave: Adolescencia temprana, recién nacido, complicaciones. (Fuente: DeCS BIREME)

\section{Newborn complications in early teenage mothers at Hospital Nacional Arzobispo Loayza from} may 2008 to may 2012

\section{ABSTRACT}

Objective: Due to the increase in fertility rate in teenagers, the interest in knowing the possible complications in the newborns also has increased. Several studies discovered that pregnancy in extreme ages is considered a risk factor for complications in newborns. Other studies have not identified a correlation between teenage pregnancy and complications in newborns. The purpose of this study is to analyze the association of complications in newborns of early teenage mothers (10 to 14 years old) compared to the newborns of adult mothers ( 20 to 35 years old).

Material and Methods: Analytic, retrospective cohort study. The population is the total amount of newborns of early teens plus the same amount of mothers between the ages of 20 and 35 years.

Results: From the total of newborns (16 601), $0.37 \%$ were part of the early teenagers group. $87.1 \%$ of the early teens and $72.6 \%$ of the adults mothers group were born within the normal weight range. $90.3 \%$ of both groups were born to term. $82.3 \%$ and $69.4 \%$ respectively, were vaginal deliveries. $95.2 \%$ and $96.8 \%$ respectively, presented jaundice. Sepsis was present in $93.5 \%$ and $96.8 \%$ respectively. Respiratory complications present in $96.8 \%$ of both groups. Mechanic complications in $90.3 \%$ and $95.2 \%$, respectively.

Conclusions: The frequency of newborns of early teens was less than national reports. There is no difference between the complications of newborns of early teenagers compared to the newborn of mothers between 20 and 35 years old.

(Horiz Med 2015; 15(1): 14-20)

Key words: Early teenagers, newborns, complications (source: MeSH NLM)

Médico Cirujano

Médico de la Clínica Ricardo Palma, Lima- Perú 


\section{INTRODUCCIÓN}

La OMS define la adolescencia como "la etapa que transcurre entre los 10 y 19 años, considerándose dos fases, la adolescencia temprana 10 a 14 años y la adolescencia tardía 15 a 19 años” $(1,2)$.

La edad media del período de la menarquia es de 11 años, aunque esta cifra varía según el origen étnico y el peso. El promedio de edad de la menarquia ha disminuido y continúa haciéndolo.

Esta disminución es un factor importante que ha permitido la aparición de embarazos a edades más tempranas y depende, por tanto, no solo de factores biológicos sino de factores sociales y personales.

Según cifras del MINSA, 3 de cada 10 mujeres sexualmente activas son adolescentes. Por otro lado, el uso de métodos anticonceptivos, es menor en este grupo etáreo; esto ha llevado a un aumento en la tasa de fecundidad en las adolescentes (3).

En países desarrollados los datos de embarazos en la adolescencia confirman una relación con los niveles educativos más bajos, las mayores tasas de pobreza y otras situaciones de inestabilidad familiar y social.

La adolescente peruana embarazada se caracteriza por tener menor grado de instrucción, inestabilidad conyugal y alta dependencia económica. Este hallazgo es común en América Latina (4-10).

Varios estudios, en distintos lugares del mundo, han concluido que el embarazo en las edades extremas del periodo reproductivo, son un factor de riesgo para complicaciones en los recién nacidos ( $\mathrm{RN})$.

Si bien la posibilidad de parto prematuro en las pacientes adolescentes está ligado a distintos factores como el bajo nivel socioeconómico, las poblaciones en algunos estudios realizados en similares características, demuestran que el riesgo de parto prematuro es mayor en las pacientes adolescentes embarazadas $(11,12)$

En la mayoría de estudios, se encuentra una frecuencia mayor de RN de bajo peso al nacimiento (principal riesgo del RN de madre adolescente) con cifras del $18 \%(12-17)$.

En Sudáfrica, se encontró que no existía asociación entre la adolescencia y mayor presentación de partos pretérminos, bajo peso al nacer, muerte a la hora del parto y puntaje APGAR. La muerte fetal y parto por cesárea fue significativamente menor en los RN de madres adolescentes tempranas (18).

En cuanto a características y complicaciones en el neonato: tipo de parto, el peso para la edad gestacional, APGAR y parto prematuro; no se encontró relación entre la edad de la madre y aumento de riesgo en las características del RN (19).

Acerca del Control Pre Natal, el Ministerio de Salud (MINSA) lo define como "la vigilancia y evaluación integral de la gestante y el feto que realiza el profesional de salud con el objetivo de lograr el nacimiento de un recién nacido sano, sin deterioro de la salud de la madre" (20).

En los últimos años ha existido polémica para definir el número óptimo de controles prenatales (CPN) y la frecuencia $(21,22)$. En el año 2007 la OMS concluyó que los embarazos de bajo riesgo obstétrico podrían tener cuatro CPN (23). El MINSA considera una gestante controlada si tiene al menos seis CPN, distribuidos de la siguiente manera: Dos antes de las 22 semanas, el tercero entre la 22 y 24 , el cuarto entre la 27 a 29, el quinto entre la 33 y 35 y el sexto entre la 37 y la última semana de gestación (20). Según el informe de la encuesta demográfica ENDES Continua 2009, 94,5\% de mujeres tuvieron algún CPN por personal de salud; pero, en la selva fue $83,5 \%$ (24).

El objetivo de este trabajo fue analizar la relación de complicaciones en RN de madres adolescentes tempranas.

\section{MATERIAL Y MÉTODOS}

Estudio analítico, de tipo cohorte retrospectivo, tomando como población a los recién nacidos de madres adolescentes tempranas y de madres entre 20 a 35 años en el servicio de Neonatología del Hospital Nacional Arzobispo Loayza, durante mayo del año 2008 hasta mayo del año 2012. 
Se recopiló información de la base de datos del Servicio de Neonatología, para luego realizar la revisión de Historias Clínicas de los pacientes seleccionados.

Criterios de inclusión: pacientes hospitalizados en el servicio de Neonatología del HNAL entre mayo del 2008 y mayo del 2012 que cuenten con historias clínicas completas para la recolección de datos necesarios.

Los que fuesen hijos de madres adolescentes tempranas (10 a 14 años) y de madres entre 20 a 35 años. Fueron excluidos los neonatos de madres en rango de edades fuera de las anteriormente descritas.

Por el número reducido de $\mathrm{RN}$ de madres adolescentes tempranas, se tomó la totalidad de pacientes.

Luego, se tomó un número equivalente de $\mathrm{RN}$ de madres entre 20 a 35 años, de manera aleatoria.

Los datos, fueron trabajados en Microsoft Excel 2010 y luego exportados al programa IBM SPSS Statistics 20.0, donde se realizaron las tablas para luego ser utilizadas en el análisis de datos.

Posteriormente, se utilizó la prueba del chi cuadrado para evaluar la asociación entre las variables según el grupo etáreo de las madres. Se calculó el Riesgo Relativo (RR) junto al Intervalo de Confianza (IC) al $95 \%$. Por último, se graficaron y analizaron los resultados encontrados.

\section{RESULTADOS}

El número total de $\mathrm{RN}$ en el periodo de tiempo del estudio fue de 16,601. El número total de RN de madres adolescentes tempranas en el mismo periodo fue de 62 .

De la totalidad de los pacientes en el estudio, 57 (29 del grupo de adolescentes tempranos y 28 del grupo control) fueron de sexo femenino y 67 (33 del grupo de adolescentes tempranos y 34 del grupo control) de sexo masculino.
Según la edad gestacional al momento de nacer, en los dos grupos predominaron los RN a término, llegando a 56 pacientes (90.3\%) en ambos grupos. Los RN pretérmino, fueron $5(8.1 \%)$ en el grupo de adolescentes tempranas y 4 (6.5\%) en el segundo grupo. 1 (1.6\%) de los RN de madres adolescentes tempranas fueron postérmino, 2 (3.2\%) se dieron en el segundo grupo.

Peso en RN de madres adolescentes: 5 (8.1\%) tuvieron bajo peso al nacer (BPN), 1 (1.6\%) con extremado bajo peso al nacer, 2 (3.2\%) macrosómicos, 0 muy bajo peso al nacer (MBPN) y 54 (87.1\%) peso normal. En el grupo de recién nacidos de madres entre 20 a 35 años; 5 (8.1\%) de BPN, 1 (1.6\%) de EBPN, $5(8.1 \%)$ con macrosomía, 1 (1.6\%) de MBPN y $50(80.6 \%)$ de peso normal.

Se presentaron, en el grupo de 11 a 14 años, 11 (17.7\%) cesáreas y 51 (82.3\%) partos vaginales. En el segundo grupo de recién nacidos, se presentaron 19 (30.6\%) cesáreas y 43 (69.4\%) partos vaginales. (Tabla 1).

Test de Apgar al minuto y a los 5 minutos: en el grupo de RN de madres adolescentes tempranas fueron $57(91.9 \%)$ con un puntaje mayor o igual a 7 al minuto y $60(96.8 \%)$ a los 5 minutos. Los resultados en el segundo grupo de RN: 59 (95.2\%) fueron mayor igual a 7 al minuto y 61 (98.4\%) a los 5 minutos.

Controles pre natales: considerando 6 o más controles como adecuados y menos como no adecuado; en madres adolescentes tempranas se dieron adecuadamente en 42 (67.7\%) mientras que en las madres de 20 a 35 años, se vio en 50 (80.6\%) de las mismas.

Sólo falleció un RN, del grupo de madres adolescentes tempranas.

Acerca de la presentación de ictericia patológica en los recién nacidos, se encontró que la mayoría de los dos grupos no presentaron este cuadro; 59 (95.2\%) del grupo de recién nacidos de madres adolescentes tempranas y 60 (96.8\%) de recién nacidos de madres entre 20 a 35 años. 
Tabla 1. Inducción del trabajo de parto en CHPR. $\mathrm{n}=200$ casos

\begin{tabular}{|c|c|c|c|c|c|c|c|c|c|c|}
\hline & & \multicolumn{2}{|c|}{$\begin{array}{c}\text { Adolescentes } \\
\text { Tempranas } \\
(n=62)\end{array}$} & \multicolumn{2}{|c|}{$\begin{array}{c}20 \text { a } 35 \\
\text { años } \\
(n=62)\end{array}$} & \multirow{2}{*}{$\begin{array}{c}\text { Chi } \\
\text { cuadrado }\end{array}$} & \multirow{2}{*}{$\begin{array}{c}\text { Grado } \\
\text { de Libertad }\end{array}$} & \multirow[b]{2}{*}{$p$} & \multirow[b]{2}{*}{$\mathbf{R R}$} & \multirow[b]{2}{*}{ IC al $95 \%$} \\
\hline \multicolumn{2}{|c|}{ Característica } & & & & & & & & & \\
\hline \multirow{3}{*}{$\begin{array}{l}\text { Edad } \\
\text { Gestacional }\end{array}$} & Postérmino & 1 & 1.6 & 2 & 3.2 & \multirow{3}{*}{0.444} & \multirow{3}{*}{2} & \multirow{3}{*}{0.801} & & \\
\hline & Pretérmino & 5 & 8.1 & 4 & 6.5 & & & & & \\
\hline & A término & 56 & 90.3 & 56 & 90.3 & & & & & \\
\hline \multirow{5}{*}{$\begin{array}{l}\text { Peso } \\
\text { al nacer }\end{array}$} & BPN & 5 & 8.1 & 5 & 8.1 & \multirow{5}{*}{2.440} & \multirow{5}{*}{4} & \multirow{5}{*}{0.655} & & \\
\hline & MBPN & 0 & 0 & 1 & 1.6 & & & & & \\
\hline & EBPN & 1 & 1.6 & 1 & 1.6 & & & & & \\
\hline & Normal & 54 & 87.1 & 50 & 80.6 & & & & & \\
\hline & Macrosomía & 2 & 3.2 & 5 & 5.6 & & & & & \\
\hline \multirow{2}{*}{$\begin{array}{l}\text { Tipo de } \\
\text { parto }\end{array}$} & Césarea & 11 & 17.7 & 19 & 30.6 & \multirow{2}{*}{2.814} & \multirow{2}{*}{1} & \multirow{2}{*}{0.093} & \multirow{2}{*}{0.676} & \multirow{2}{*}{$0.408-1.120$} \\
\hline & Vaginal & 51 & 82.3 & 43 & 69.4 & & & & & \\
\hline \multirow{2}{*}{$\begin{array}{l}\text { APGAR al } \\
\text { minuto }\end{array}$} & $>7$ & 57 & 91.9 & 59 & 95.2 & \multirow{2}{*}{0.534} & \multirow{2}{*}{1} & \multirow{2}{*}{0.465} & \multirow{2}{*}{1.272} & \multirow{2}{*}{$0.721-2.244$} \\
\hline & $<7$ & 5 & 8.1 & 3 & 4.8 & & & & & \\
\hline \multirow{2}{*}{$\begin{array}{l}\text { APGAR a } \\
\text { los } 5 \text { minutos }\end{array}$} & $>7$ & 60 & 96.8 & 61 & 98.4 & \multirow{2}{*}{0.342} & \multirow{2}{*}{1} & \multirow{2}{*}{0.559} & \multirow{2}{*}{1.344} & 0 592-3053 \\
\hline & $<7$ & 2 & 3.2 & 1 & 1.6 & & & & & \\
\hline CPN & SI & 42 & 67.7 & 50 & 80.6 & & & & 1369 & 066-1-1941 \\
\hline CPN & NO & 20 & 32.3 & 12 & 19.4 & 2.696 & 1 & 0.101 & 1.369 & $0.966-1-1.941$ \\
\hline & AEG & 53 & 85.5 & 45 & 72.6 & & & & & \\
\hline $\begin{array}{l}\text { geso pacional eara } \\
\text { gestaciona }\end{array}$ & GEG & 3 & 4.8 & 8 & 12.9 & 3.735 & 4 & 0.443 & & \\
\hline & PEG & 6 & 9.7 & 9 & 14.5 & & & & & \\
\hline
\end{tabular}

Sepsis neonatal: en el grupo de RN de madres adolescentes tempranas, se encontraron 4 casos $(6,5 \%)$, mientras que en el segundo grupo se encontraron 2 casos (3.2\%).

Dentro de las complicaciones respiratorias en el $\mathrm{RN}$, se incluyó la enfermedad de membrana hialina, neumotórax del RN y apnea. Dos pacientes (96.8\%) de cada uno de los dos grupos presentaron dichas complicaciones.

Hipoglicemia en el RN: sólo se presentaron dos (3.2\%) casos en los RN de madres adolescentes tempranas.

Complicaciones mecánicas y/o traumáticas: cefalohematoma, caput succedanum y fractura de clavícula. Se encontraron en 6 (9.7\%) RN del primer grupo y 3 (4.8\%) del segundo grupo.

Ingresos a Unidad de Cuidados Intensivos y/o Intermedios: se encontraron 2 (3.2\%) pacientes del grupo de RN de madres adolescentes tempranas y 1 (1.6\%) de los RN de madres entre 20 a 35 años.
Acerca del peso para la edad gestacional, se encontraron en el primer grupo: 53 (85.5\%) Adecuados para Edad Gestacional; 3 (4.8\%) Grande para Edad Gestacional; 6 (9.6\%\%) de Pequeños para Edad Gestacional y en el otro grupo, 45 (72.9\%) Adecuados para Edad Gestacional; 8 (12.9\%) Grande para Edad Gestacional; 9 (14.4\%) de Pequeños para Edad Gestacional.

\section{DISCUSIÓN}

Estudios previos han demostrado el aumento de frecuencia de parto durante la adolescencia oscilando entre 7 y $25 \%$ siendo mayor en los países en desarrollo $(25,27)$. Por tal motivo, es que la atención para las madres adolescentes se ha convertido en prioridad y se está dando asistencia para que tenga una mayor accesibilidad a los servicios de salud, implementando estrategias, fortaleciendo programas y sacando el financiamiento adecuado $(27,29)$.

Se ha comprobado en diversos estudios la importancia y utilidad de los controles prenatales para disminuir la morbimortalidad materna e 
Tabla 2. Inducción del trabajo de parto en CHPR. n=200 casos

\begin{tabular}{|c|c|c|c|c|c|c|c|c|c|c|}
\hline \multirow[b]{2}{*}{ Compilación } & & \multicolumn{2}{|c|}{$\begin{array}{c}\text { Adolescentes } \\
\text { Tempranas } \\
(n=62)\end{array}$} & \multicolumn{2}{|c|}{$\begin{array}{c}20 \text { a } 35 \\
\text { años } \\
(n=62)\end{array}$} & \multirow[b]{2}{*}{$\begin{array}{c}\text { Chi } \\
\text { cuadrado }\end{array}$} & \multirow[b]{2}{*}{$\begin{array}{c}\text { Grado } \\
\text { de Libertad }\end{array}$} & \multirow[b]{2}{*}{$p$} & \multirow[b]{2}{*}{$\mathbf{R R}$} & \multirow[b]{2}{*}{ IC al $95 \%$} \\
\hline & & $\mathrm{n}$ & $\%$ & $\mathbf{n}$ & $\%$ & & & & & \\
\hline \multirow[t]{2}{*}{ Muerte } & No & 61 & 98.4 & 62 & 100 & \multirow{2}{*}{1.008} & \multirow{2}{*}{1} & \multirow{2}{*}{0.315} & \multirow{2}{*}{0.496} & \multirow{2}{*}{$0.415-0.593$} \\
\hline & $\mathrm{Si}$ & 1 & 1.6 & 0 & 0 & & & & & \\
\hline \multirow[t]{2}{*}{ Ictericia } & No & 59 & 95.4 & 60 & 96.8 & \multirow{2}{*}{0.208} & \multirow{2}{*}{1} & \multirow{2}{*}{0.648} & \multirow{2}{*}{0.826} & \multirow{2}{*}{$0.395-1.729$} \\
\hline & $\mathrm{Si}$ & 3 & 4.8 & 2 & 3.2 & & & & & \\
\hline \multirow[t]{2}{*}{ Sepsis } & No & 58 & 93.5 & 60 & 96.8 & \multirow{2}{*}{0.701} & \multirow{2}{*}{1} & \multirow{2}{*}{0.403} & \multirow{2}{*}{0.737} & \multirow{2}{*}{$0.407-1.336$} \\
\hline & $\mathrm{Si}$ & 4 & 6.5 & 2 & 3.2 & & & & & \\
\hline \multirow[t]{2}{*}{ Respiratorias } & No & 60 & 96.8 & 60 & 96.8 & \multirow{2}{*}{0.000} & \multirow{2}{*}{1} & \multirow{2}{*}{1.000} & \multirow{2}{*}{1.000} & \multirow{2}{*}{$0.369-2.708$} \\
\hline & $\mathrm{Si}$ & 2 & 3.2 & 2 & 3.2 & & & & & \\
\hline \multirow{2}{*}{ Hipoglicemia } & No & 60 & 96.8 & 62 & 100 & \multirow{2}{*}{2.003} & \multirow{2}{*}{1} & \multirow{2}{*}{0.154} & 0492 & $0411-0589$ \\
\hline & $\mathrm{Si}$ & 2 & 3.2 & 0 & 0 & & & & 0.492 & $0.411-0.589$ \\
\hline Mecánicas/Traumáticas & No & 56 & 90.3 & 59 & 95.2 & 1708 & 1 & 0.299 & 0.730 & $0.444-1.203$ \\
\hline mecamicast itraumaticas & $\mathrm{Si}$ & 6 & 9.7 & 3 & 4.8 & 1.100 & 1 & 0.299 & 0.130 & $0.444-1.203$ \\
\hline Ingreso a UCIUCIN & No & 60 & 96.8 & 61 & 98.4 & 0342 & 1 & 0.552 & 0744 & $0.328-1689$ \\
\hline & $\mathrm{Si}$ & 2 & 3.2 & 1 & 1.6 & & & & & \\
\hline
\end{tabular}

infantil (30). Es alarmante ver el número de madres de adolescentes tempranas (32.4\%) que no cumplen con un número adecuado de controles prenatales, al igual que las madres de 20 a 35 años (19.4\%). Similar resultado se encontró en un estudio realizado en el Hospital Nacional Cayetano Heredia, en el que solo el $66.05 \%$ de las pacientes obtuvieron más de 6 controles prenatales, cifra que nos hace reflexionar y cuestionar qué es lo que está pasando y cómo poder mejorarlo (30).

Otra cifra que llama la atención es el alto número de cesáreas, las cuales se dieron en $30,6 \%$ de las madres entre 20 a 35 años y $17.7 \%$ en madres adolescentes tempranas, lo que demuestra la tendencia aumentado por la elección de este método para finalizar el embarazo. Si bien con el pasar del tiempo se ha mejorado mucho en la técnica, este procedimiento conlleva a un mayor riesgo de morbimortalidad en la madre y el recién nacido.

La frecuencia de partos de adolescentes tempranos en el Hospital Loayza en este periodo de tiempo fue de $0.37 \%$, por debajo de la estadística nacional $(0,99 \%)$. En un estudio realizado en el Hospital Nacional Daniel Alcides Carrión, con una población similar a la estudiada, se encontró una frecuencia de $1.42 \%(31,32)$.
En este estudio no se encontró asociación entre las características y complicaciones de los neonatos con relación al grupo etáreo materno.

A pesar que hayestudios que demuestran locontrario, vemos que el número de complicaciones entre los recién nacidos de madres adolescentes tempranas y los recién nacidos de madres entre 20 a 35 años son similares. En un estudio realizado en el año 2000 en el Perú, el cual comparaba complicaciones entre pacientes adolescentes tempranas y tardías (materna y de los recién nacidos), concluyeron que mientras más adolescentes sean las madres, tienen mayor probabilidad de presentar parto distócico, parto pretérmino, y sus productos bajo peso al nacer, depresión neonatal y complicaciones perinatales (31).

En un estudio publicado en junio del 2010, realizado en Sudáfrica, el cual compara los resultados tanto perinatales como obstétricos en adolescentes tempranas, tardías y adultas mujeres, concluye que en la parte obstétrica, no existe riesgo extra en los embarazos tempranos, en cuanto a los resultados en los recién nacidos, se encontró que no había relación (18).

En otro estudio publicado en enero del 2012, en donde comparan resultados en la madre y recién 
nacidos de grupos de adolescentes, adultas y de edad avanzada, se demostró que el grupo de mayor riesgo para presentar complicaciones tanto maternas como neonatales fue el grupo de madres en edad avanzada (32).

Existe otro estudio, el cual concluye que el ser adolescente, incluso es un factor protector que disminuye el riesgo de terminación de parto por cesárea (RR 0.85 IC95\% 0, 78-0.93). En contraste, concluyen que los recién nacidos de madres adolescentes tienen un mayor riesgo de presentar prematurez, bajo peso y de ser pequeños para edad gestacional, en comparación con los neonatos de madres de 15 a 29 años (34).

En conclusión, en el periodo de mayo del 2008 a mayo 2012 en el Hospital Nacional Arzobispo Loayza, no existió diferencia tanto en las características como en las complicaciones de los recién nacidos de madres adolescentes tempranas en relación a los recién nacidos de madres entre 20 a 35 años. Se debería tomar en cuenta esta situación y posiblemente, apoyado con estudios posteriores que lo avalen, dejar de tomar a estas pacientes como pacientes con Alto Riesgo Obstétrico.

Por otra parte, el estudio concluye que la frecuencia de recién nacidos de madres adolescentes tempranas es menor a la estadística nacional, mostrando posiblemente una mayor información y educación en las pacientes en el HNAL en el período indicado.

Si bien se ha demostrado que no hay asociación a corto plazo, sería importante evaluar las complicaciones a largo plazo en los hij os de madres adolescentes tempranas. Otra recomendación importante seria, en base al actual trabajo, comparar con una población mayor, ampliando así el periodo de estudio.

\section{REFERENCIAS BIBLIOGRÁFICAS}

1. INEI. Sistema de Consulta de Principales Indicadores Demográficos, Sociales y Económicos. Perú: Censos Nacionales 2007, XI de Población Y VI de Vivienda. (2007). Consultado el 27 de diciembre de 2011.

2. Organización Panamericana de la Salud. Salud del Adolescente. OPS/OMS Washington.1995.
3. http://www.minsa.gob.pe/portada/especiales/2010/ embarazoadolescente/ archivos/ estadisticas. pdf

4. Vigil P, Arias T, Lezcano G, Caballero L, Chong J, De Mendieta A, et al. Embarazo en adolescentes en la República de Panamá. Rev Obstet Ginecol Venez 2007;67:73-7.

5. Doig J. Indicadores perinatales en hijos de madres adolescentes del Instituto Especializado Materno Perinatal durante el año 2003. Rev Per Pediat 2006;59:6-11.

6. Sandoval J, Mondragón F, Ortiz M. Complicaciones materno perinatales del embarazo en primigestas adolescentes: Estudio caso-control. Rev Per Ginecol Obstet 2007;53:28-34.

7. León $\mathrm{P}$, Minassian $\mathrm{M}$, Borgoño R, Bustamante $\mathrm{F}$. Embarazo adolescente. Universidad de Chile. Rev Pediat Electrónica 2008; 5:42-51.

8. González A, Alonso R, Gutiérrez A, Campo A. Estudio de gestantes adolescentes y su repercusión en el recién nacido en un área de salud. Rev Cubana Pediatr 2000; 72:54-9

9. León R, Méndez D, Ng Wong Y, Parra L, Peña J, Rísquez A. Embarazo adolescente como factor de riesgo en la salud infantil, Ambulatorio El Valle, Caracas 2007. Ciencia e Investigación Médica Estudiantil Latinoamericana 2009; 14:42-7.

10. Quezada C, Delgado A, Arroyo L, Díaz M. Prevalencia de lactancia y factores socio demográficos asociados en madres adolescentes. INPER Isidro Espinoza de los Reyes México 2008; 65:19-25.

11. Vera G, Gallegos MS, Varela M.Salud mental y embarazo en adolescentes. Contexto de la gestación y consecuencias biográficas tempranas. Rev Méd Chile 1999; 127: 437-43.

12. Padilla de Gil.Aspectos médicos y sociales de la maternidad en la adolescencia. Rev Sogia 2000; 7(1): 16-25.

13. Ruoti A.Patología obstétrica en la adolescente embarazada. Rev Sogia 1994; 1(2): 70

14. Stewart A. Adolescent pregnancy. Lancet 1990; 335(8685): 356.

15. T J Cantor. Adolescent pregnancy. Lancet 1989; 2(8675): 1308-9

16. Kumbi S, Isehak A.Obstetric outcome of teenage pregnancy in north western Ethiopia. East Afr Med J 1999; 76(3): 138-40.

17. Thame M, Wilks R, Matadial L, Forrester TE. A comparative study of pregnancy outcome in teenage girls and mature women. West Indian Med J 1999; 48(2): 69-72.

18. Hoque. Comparación de resultados perinatales y obstétricos en adolescentes tempranas, tardías y adultos muj eres embarazadas de Sudáfrica rural. 2010. East Afr J Public Health.

19. Díaz, A. Sanhueza, P. Riesgos obstétricos en el embarazo adolescente: estudio comparativo de resultados obstétricos y perinatales con pacientes embarazadas adultas. Rev chil obstet ginecol 2002; 67(6): 481-487

20. Ministerio de Salud. Guías Nacionales de Atención Integral de la Salud Sexual y Reproductiva. Lima, Perú: MINSA. enero del 2011.

21. Khan-Neelofur D, Gulmezoglu M, Villar J. Who should provide routine antenatal care for low-risk women, and how often? A systematic review of randomised controlled trials. Paediatric and Perinatal Epidemiology 1998; 12: 7-26. 
22. McCaw-Binns A, La Grenade J, Ashley D. Under-users of antenatal care: a comparison of non-attenders and late attenders for antenatal care, with early attenders. SocSci Med 1995; 40(7): 03-12.

23. Organización Mundial de la Salud. Ensayo clínico aleatorizado de control prenatal de la OMS: Manual para la puesta en práctica del nuevo modelo de control prenatal. Agosto del 2010.

24. Instituto Nacional de Estadística e Informática. Encuesta Demográfica y de Salud Familiar - ENDES Continua 2009. Perú Lima, Mayo 2010.

25. Bachman C. Adolescencia. Riesgo reproductivo.Omega S. A. Lima 1989.

26. Kondamudi VK Adolescents pregnancy in Grenada. Ann-TropPaediatr 1993; 13 (4): 379-83.

27. Salvador J, Maradiegue E. Gestación en adolescentes. Hospital Nacional Cayetano Heredia. Ginecol Obstet Perú 1995; 41(3).

28. Klein JD, Slap GB. Adolescents and access to health care. Bull 70 (3): 219-35.

29. Brindis CD. What it will take placing adolescents on the American National Agenda for the 1990s. J Adolesc Health. 1993; 14 (7): 527. 30.

\section{Fuentes de financiamiento}

Este estudio ha sido autofinanciado por el autor.

\section{Conflicto de interés}

\section{El autor declara no tener ningún conflicto de} interés
30. Arispe, Salgado. Frecuencia de control prenatal inadecuado y de factores asociados a su ocurrencia. Rev Med Hered 2011; 22(4): 169175.

31. Jorge Yu-Tang. Complicaciones Perinatales y Vía de Parto en Recién Nacidos de Madres Adolescentes Tempranas y Tardías entre 1995 y 1997. 2000. Revista Anales de Medicina de la Facultad de Medicina Humana de la Universidad Mayor de San Marcos.

32. Diana Huanco A. PhD, Manuel Ticona R. PhD, Maricarmen Ticona V. Flor Huanco A. Frecuencia y repercusiones maternas y perinatales del embarazo en adolescentes atendidas en hospitales del Ministerio de Salud del Perú. 2008. Rev Chil Obstet Ginecol 2012; 77(2): 122 128

33. Kuyumcuoglu. La comparación de los factores de riesgo de resultados perinatales adversos en los embarazos de adolescentes y de embarazos de edad avanzada. 2012. Ginekol Pol.

34. Jairo Amaya, Claudia Borrer y Santiago Ucrós. Estudio Analítico del Resultado del Embarazo en Adolescentes y Mujeres de 20 a 29 años en Bogotá en el año 2005. Revista Colombiana de Obstetricia y Ginecología.

\section{Correspondencia:}

Alonso F. La Rosa de los Ríos

Dirección: Calle Centenario 184 - La Molina - Lima

- Perú

Teléfono: 013493120

Correo electrónico: alonso_larosa@usmp.pe 